\title{
Las Wikis como Recurso Didáctico para la Enseñanza en Asignaturas del Área de Ciencias Experimentales
}

Ana M. Díez-Pascual ${ }^{\mathrm{a}}$, Pilar García Díaz ${ }^{\mathrm{b}}$, Rafael Cambralla Diana ${ }^{\mathrm{b}}$ y Miguel Ángel Ortega Núñez ${ }^{c}$

a Departamento de Química Analítica, Química Física e Ingeniería Química, am.diez@uah.es. bepartamento de Teoría de la Señal y Comunicaciones, pilar.garcia@uah.es; rafael.cambralla@uah.es; ${ }^{c}$ Departamento de Medicina y Especialidades Médicas. Universidad de Alcalá

\begin{abstract}
A wiki is a collaborative website that provides development and modification of its content and structure to every member in an interactive, easy and fast way. It is a simple and valuable tool for the teaching-learning process that allows both teachers and students to collaborate in the generation of knowledge. This paper describes the teaching experience in the implementation of a wiki as a voluntary activity in a practical subject of the first course of the degree in Chemistry. This methodology has been used for the elaboration of a bilingual glossary with the conceptual terms learned during the classes. The didactic strategy has been positive, although the percentage of participation in the wiki has been scarce. Only the students most committed with the subject were involved in the construction of the wiki. Therefore, it is necessary to introduce modifications in the approach of the activity in order to obtain more satisfactory results.
\end{abstract}

Keywords: Wiki, competences, methodology, collaborative work, teachinglearning, glossary of terms, feedback

\section{Resumen}

Una wiki es un sitio web colaborativo donde sus miembros pueden crear y modificar los contenidos y su estructura de forma interactiva, fácil y rápida. Es una herramienta sencilla y valiosa para el proceso de enseñanzaaprendizaje que permite tanto a los docentes como a los alumnos colaborar en la generación de conocimiento. Este trabajo recoge la experiencia docente en la implementación de una wiki como actividad voluntaria en una asignatura práctica del primer curso del Grado en Química. Esta metodología se ha utilizado para la elaboración de un glosario bilingüe con los términos conceptuales aprendidos durante las clases presenciales. La estrategia didáctica ha resultado positiva, si bien el porcentaje de participación en la misma ha sido escaso. Solo los alumnos más comprometidos con la asignatura se involucraron en la construcción de la wiki. Por tanto, se requiere introducir modificaciones en el planteamiento de la actividad para conseguir resultados más satisfactorios.
} 
Las Wikis como Recurso Didáctico para la Enseñanza en Asignaturas del Área de Ciencias Experimentales

Palabras clave: wiki, competencias, metodología, trabajo colaborativo, enseñanza-aprendizaje, glosario de términos, feedback

\section{Introducción}

Una wiki es un sitio web cuyas páginas pueden ser editadas por múltiples usuarios a través del navegador, entendiéndose por navegador un programa que permite visualizar la información que contiene una página web (ya esté alojada en un servidor dentro de la world wide web o en uno local). En la wiki los usuarios pueden crear y modificar los contenidos, el formato y su estructura de forma interactiva, fácil y rápida (Cabero y Llorente, 2007); dichas facilidades la convierten en una herramienta eficaz para la escritura colaborativa, donde los miembros cooperan en el desarrollo de contenidos sobre una temática concreta. Las wikis pertenecen al mismo grupo de herramientas para uso compartido de contenidos web que los blogs o los foros (López Sastre y Pérez Köhler, 2007), si bien hay importantes diferencias entre estos tres sitios web. Un blog suele tener un único autor o editor, el cual incluye a modo de diario personal contenidos de su interés, que son comentados por los lectores. El autor administra su espacio blog y tiene la capacidad de hacer visible o no los comentarios referentes a sus contenidos. Como diferencia fundamental, en una wiki cualquier usuario puede acceder a la edición de los bloques de contenidos y participar en la discusión comentando dichos bloques. Por otro lado, un foro es un sitio de discusión online asincrónico donde los usuarios publican mensajes relacionados con un tema, fomentando así el debate espontáneo. Se parece a la parte de discusión del blog o de la wiki, pero estos últimos tienen la función adicional de recopilación de contenidos.

Las wikis aplicadas en docencia permiten que los estudiantes muestren los hitos alcanzados a lo largo de su proceso de aprendizaje (García Díaz, 2016). Además contribuyen positivamente al proceso de enseñanza-aprendizaje desempeñando una serie de funciones de gran importancia como son: la motivación y persuasión del estudiante para realizar actividades de la asignatura, el apoyo al aprendizaje autónomo, el establecimiento de una vía de comunicación directa entre el profesor y los alumnos fuera del entorno del aula y la evaluación formativa de carácter continuo (Ortega Núñez, 2016).

Los sitios de alojamiento web más utilizados en el área de la educación para la creación de wikis son: PBworks, Wetpaint, Wikia, Google Sites y Wikispaces. Todos ellos utilizan servicios web gratuitos y un servidor remoto transparente al usuario. En la experiencia que se describe a continuación se ha empleado Wikispaces, un servicio lanzado por Tangient LLC en 2005, que fue comprado por Wikispaces TSL Educación en 2014 (ver Figura 1).

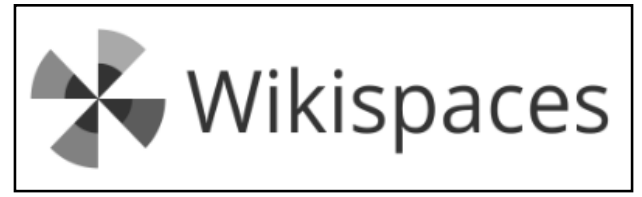

Fig. 1 Logo del servicio gratuito de Wikispaces (tomado de la página web www.wikispaces.com)

(cc) BY-NC-ND 2017, UniversitatPolitècnica de València 
Las actividades que se mencionan posteriormente están referidas a Wikispaces, si bien es posible llevar a cabo las mismas acciones con cualquier otro servicio wiki disponible actualmente. El profesor presentó a los alumnos la actividad a realizar con la wiki, con el fin de que comprendieran el uso de la aplicación, el objetivo de la actividad y su dinámica (López Sastre, 2007). La actividad se planteó dentro de la asignatura "Operaciones Básicas de Laboratorio", del primer curso del Grado en Química. Debido al carácter experimental de la asignatura, la actividad se consideró totalmente voluntaria, con un posible efecto positivo sobre la calificación final en la asignatura. La experiencia descrita en este documento es un comienzo de la reflexión en la metodología antes de incluirlo como actividad obligatoria en la asignatura.

\section{Objetivos}

- Analizar el potencial de las wikis en el contexto de la educación superior universitaria.

- Valorar una experiencia del uso educativo de wikis en el área de ciencias experimentales.

- Favorecer el trabajo tanto autónomo como colaborativo entre los alumnos. Ser capaz de coordinarse, organizar su tiempo y dividir las tareas.

- Iniciar a los estudiantes en la búsqueda bibliográfica de información, aprender y actualizar conocimientos.

- Promover el uso adecuado de las nuevas tecnologías.

- Fomentar su capacidad de discusión y sentido crítico, de toma de decisiones, capacidad de selección, síntesis y sistematización de conocimientos.

\section{Desarrollo de la innovación}

La asignatura de Operaciones Básicas de Laboratorio, materia obligatoria anual de 9 créditos ECTS del primer curso del Grado en Química de la Universidad de Alcalá (curso académico 2016-2017), incluye un laboratorio específico de "Introducción a la Experimentación en Química Analítica". Dicho laboratorio consiste en clases prácticas impartidas en grupos pequeños donde el alumno desarrolla experimentos para aprender a aplicar e interpretar los principios básicos estudiados en las clases teóricas.

Esta parte de la asignatura tiene como objetivo iniciar a los estudiantes en la sistemática del proceso analítico y capacitarlos para desarrollar criterios científicos en la evaluación de resultados analíticos. Entre sus competencias específicas se encuentran fomentar el trabajo en equipo así como conocer y saber usar el instrumental y el aparataje que se utiliza habitualmente en este tipo de laboratorios. Para alcanzar estas competencias resulta fundamental la familiarización con la terminología específica del área, por lo que es muy importante realizar actividades donde se maneje dicha terminología. Un entorno adecuado para su desarrollo es una wiki donde los alumnos pueden ir revisando y editando el trabajo en grupo, lo que fomenta tanto su aprendizaje autónomo como el trabajo colaborativo. Este tipo de actividades permiten realizar tareas que no podían llevarse a cabo en clase por el límite de tiempo y carácter de la misma.

La Figura 2 muestra la página de inicio que se encuentra cualquier miembro al acceder a la wiki mediante su cuenta de Wikispaces. La wiki que aquí presentamos consta de dos secciones: 
Las Wikis como Recurso Didáctico para la Enseñanza en Asignaturas del Área de Ciencias

zona de contenidos y área de discusión o comentarios. En la primera el profesor plantea la actividad a realizar. En el área de discusión los miembros pueden insertar comentarios relacionados con la actividad. Es una zona de debate colectivo, tanto entre alumnos como entre alumnos y profesor. Es en este contexto donde el docente puede aportar ideas y comentarios que reconduzcan la elaboración de la actividad propuesta.

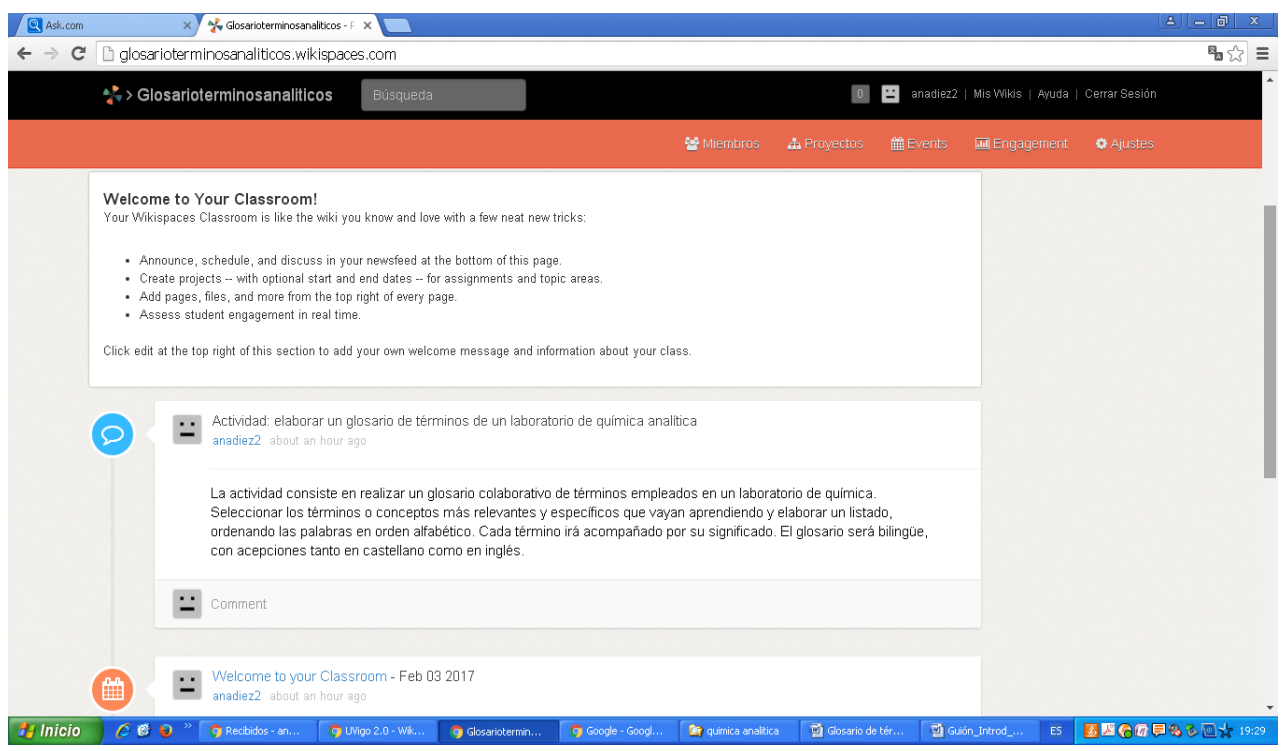

Fig. 2 Página de inicio de la wiki de la asignatura Operaciones Básicas de Laboratorio del primer curso del Grado en Química (curso académico 2016-2017).

La wiki se presentó en un seminario durante el cual se explicó su forma de utilización desde el registro en Wikispaces. La actividad consistió en realizar un glosario colaborativo entre todos los alumnos. Tenían que seleccionar los términos o conceptos más relevantes y específicos que hubiesen aprendido durante las prácticas y elaborar un listado ordenado alfabéticamente. Cada término debía ir acompañado por su significado (o significados) en el contexto de la materia estudiada, de modo similar a como se realiza en la Wikipedia. El glosario sería bilingüe, con acepciones tanto en castellano como en inglés. Para elaborar la actividad, es conveniente consultar bibliografía específica (Cuadros Rodríguez, 2013). El profesor proporciona "feedback" a través de la wiki, de manera que los alumnos puedan ir mejorando los contenidos a medida que el curso avanza.

La primera tarea consistió en realizar el listado de términos o conceptos que constituyen el glosario. Para ello, algunos alumnos presentaron una primera propuesta, y el resto del grupo añadió, modificó o suprimió los diferentes términos hasta lograr el consenso o acuerdo de todo el grupo. Se sugirió nombrar un coordinador del grupo para gestionar la dinámica grupal. La segunda tarea consistió en distribuir la redacción de los distintos conceptos en la wiki. Todos los alumnos leyeron lo redactado y aportaron, si lo consideraron oportuno, modificaciones en las definiciones de cada concepto. $\mathrm{Y}$ así sucesivamente hasta finalizar el glosario. Lo último a realizar fue dar el formato apropiado al glosario, unificar la tipografía empleada, asociarle un

(c)) BY-NC-ND 2017, UniversitatPolitècnica de València 
background común, incorporar imágenes y otros recursos gráficos. También se incorporó al final del glosario la bibliografía empleada (textos impresos y referencias en formato electrónico).

\section{Resultados}

Una vez creada la wiki "glosarioterminosanaliticos", los dos primeros alumnos que ingresaron no interpretaron bien la actividad a realizar e introdujeron solo los términos sin indicar su significado. Posteriormente otros dos miembros acometieron la elaboración del glosario de términos, indicando su acepción en los dos idiomas requeridos. Otra estudiante introdujo nuevos términos y ordenó todo el conjunto por orden alfabético. La figura 3 muestra fragmentos del glosario realizado por los estudiantes. Periódicamente, el profesor realizó algunas correciones al glosario, publicando los comentarios correspondientes.

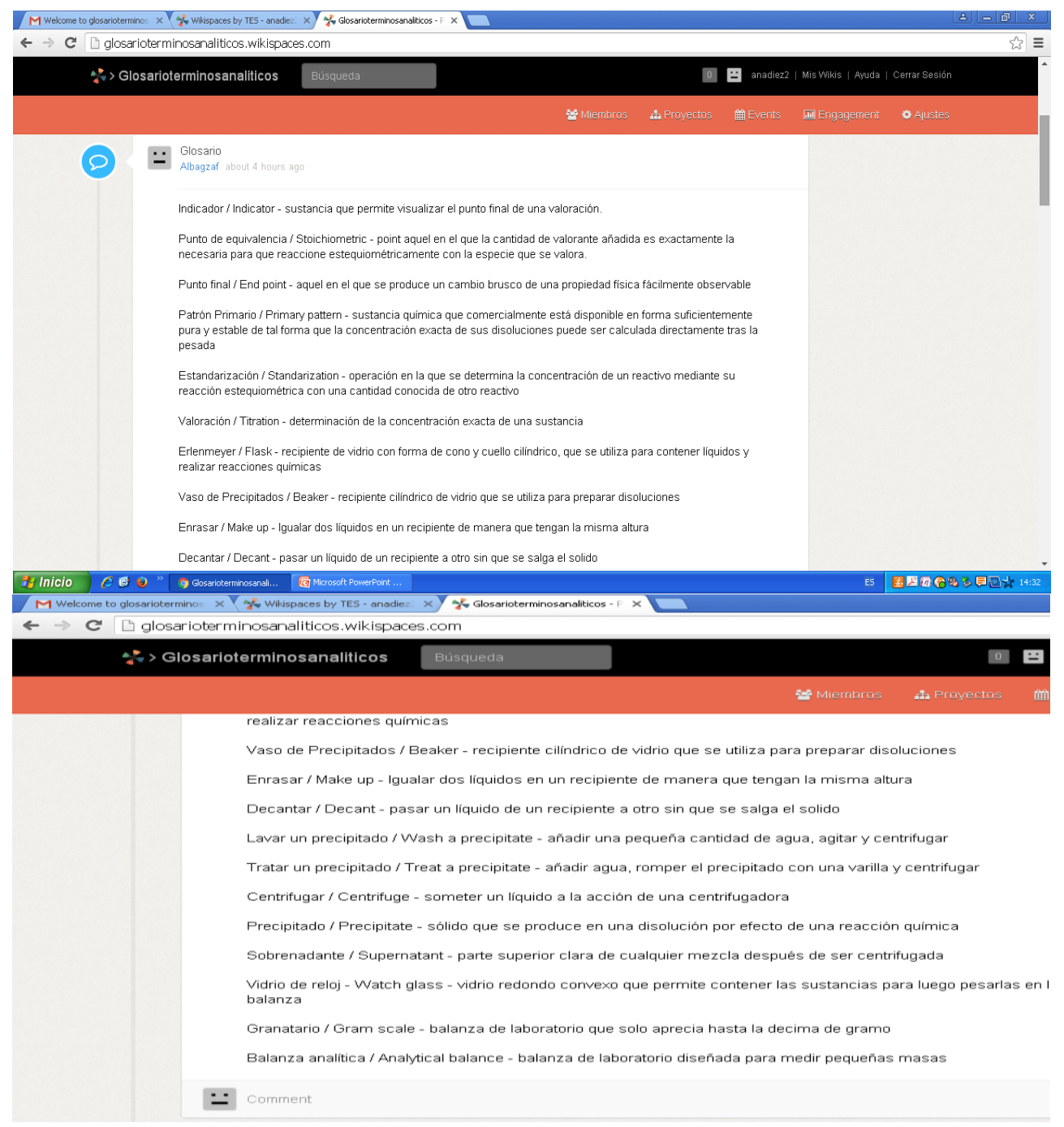

Fig. 3 Fragmentos del glosario creado por los estudiantes en la wiki 
Las Wikis como Recurso Didáctico para la Enseñanza en Asignaturas del Área de Ciencias

La figura 4 muestra los miembros que participaron en la wiki. De los 96 estudiantes de primer curso de Grado en Química, solo 5 participaron en la actividad voluntaria.

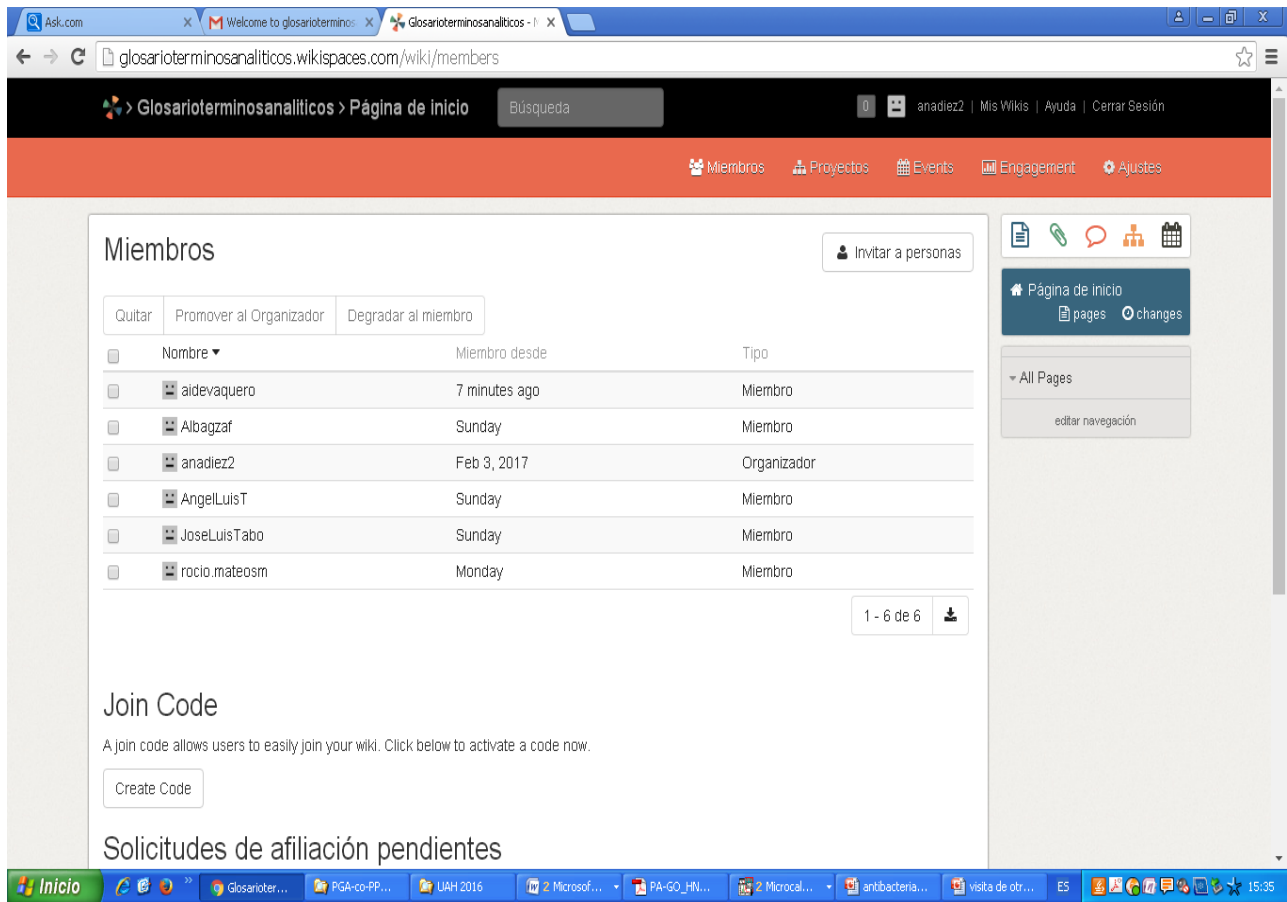

Fig. 4 Miembros que participaron en la wiki "glosarioterminosanaliticos"

La Tabla 1 recoge la estadística de participación en la wiki. En la asignatura de Operaciones Básicas de Laboratorio hay una tasa de aprobados muy baja, del 33\%. Como puede deducirse de la ¡Error! No se encuentra el origen de la referencia., la participación en la wiki fue muy escasa, solo el 5,2\% de los estudiantes contribuyeron a la actividad. Todos los miembros de la wiki aprobaron la asignatura en la convocatoria ordinaria. De hecho, tres de ellos sacaron la máxima calificación. De los alumnos aprobados, el 15,6\% corresponde a miembros de la wiki. Se analizó también el tiempo que los estudiantes dedicaron a realizar la actividad, encontrándose un rango bastante amplio, entre 1 y 6 horas dependiendo de su grado de involucración.

Tabla 1. Estadística de participación de los alumnos en la wiki y su relación con la calificación en la asignatura de Operaciones Básicas de Laboratorio.

\begin{tabular}{cc}
\hline Estadística & Alumnos (\%) \\
\hline Participación en la wiki sobre alumnos matriculados & 5,2 \\
Participación en la wiki sobre presentados a examen & 5,9 \\
Participación en la wiki sobre alumnos aprobados & 15,6 \\
Aprobados en asignatura sobre participación en la wiki & 100 \\
\hline
\end{tabular}

(cc) EY-NC-ND 2017, UniversitatPolitècnica de València 


\section{Conclusiones y perspectivas futuras}

Una conclusión inmediata del análisis de resultados es que todos los alumnos que emplearon la herramienta wiki aprobaron la asignatura. Teniendo en cuenta que se presentó como una actividad de carácter voluntario, se puede deducir que los alumnos más comprometidos con la asignatura se involucraron también en la construcción de la wiki. La no obligatoriedad de la actividad fomenta una mejor gestión del aprendizaje por parte de los alumnos, centrando su esfuerzo en el contenido de lo que escriben y no en la calificación de la actividad.

La baja participación del alumnado podría estar relacionada con el escaso tiempo del que disponen y la poca flexibilidad. La evaluación continua frecuentemente provoca solapamiento de las actividades propuestas en diferentes asignaturas, causando coincidencia prácticamente simultánea en los periodos de actividad más intensa. Con el fin de evitar esta situación se sugiere establecer criterios flexibles y consensuados con los estudiantes para los plazos y tiempos de trabajo con la wiki.

Se plantea también la posibilidad de diseñar otras actividades, de manera que los alumnos tengan la opción de elegir aquella(s) que les parezcan más interesantes para potenciar su aprendizaje. Entre ellas se encontrarían: (1) Realizar una evaluación crítica de un documento científico del área de la química analítica relacionado con los temas estudiados, el cual se colocaría en la wiki. Los alumnos deberían leer el artículo con atención y escribir una breve evaluación crítica de los objetivos del trabajo, métodos, resultados y conclusiones. Se les entregaría una lista de ayuda con preguntas relacionadas con los conceptos estudiados que aparecen en la publicación. Así, los alumnos deberán identificar los términos específicos relacionados con la materia. (2) Redacción sobre un tema de actualidad relacionado con la química analítica donde se apliquen los conocimientos y la terminología específica aprendida. Se trataría de una redacción colaborativa donde los estudiantes tendrían que coordinarse en la escritura, corrigiendo y adaptando lo que escriban los otros compañeros. Serían ellos mismos los que seleccionasen el tema a desarrollar, lo que les llevaría a explorar en la bibliografía de la materia. (3) Respuesta a preguntas concretas relacionadas con la temática de la asignatura. Esta actividad requeriría el uso del vocabulario específico y una mayor capacidad de síntesis para responder a cuestiones concretas. Serviría para afianzar los conocimientos adquiridos durante las clases, repasar los conceptos y prepararse para las diferentes pruebas de la asignatura. Algunas de las cuestiones serían en forma de ejercicios numéricos. Los alumnos responderían de forma colaborativa, y el profesor no corregiría explícitamente las respuestas, sino que las comentaría en el área de discusión.

\section{Referencias}

CABERO, J ., LLORENTE, M. (2007). "La interacción en el aprendizaje en red: uso de herramientas, elementos de análisis y posibilidades educativas" en Revista Iberoamericana de Educación a Distancia, $10,2,98-123$

CUADROS RODRÍGUEZ, L., GÁMIZ GRACIA, L., CARRASCO PANCORBO, A., RUIZ SAMBLÁS, C. (2013). "Glosario de términos analíticos". Grupo Regional Andaluz de la Real Sociedad Española de Química Analítica, 1 edición. ISBN 978-84-616-4720-0. URL: http://www.revistaquimica.cl/wp-content/uploads/2016/02/Glosario-Terminos-Analiticos.pdf 
Las Wikis como Recurso Didáctico para la Enseñanza en Asignaturas del Área de Ciencias Experimentales

GARCÍA DÍAZ, P., ORTEGA NÚÑEZ, M.A., CAMBRALLA DIANA, R., DÍEZ-PASCUAL, A. M. (2016) "Experiencia de implementación de una wiki como apoyo a la docencia presencial en enseñanzas universitarias". VIII Encuentro de Innovación en Docencia Universitaria: "Nuevas apuestas educativas : Entre la docenciapresencial y la innovación tecnológica" (25 y 26 de abril de 2016. Facultadde Biología, Ciencias Ambientales y Química. Universidad de Alcalá). Alcalá de Henares, Madrid.

LÓPEZ SASTRE, R.J. (2007). “Aprendizaje y software social: comunidades de práctica y Wikis en el EEES”. Alcalá de Henares: Servicio de Publicaciones de UAH.

LÓPEZ SASTRE, R.J., PERÉZ KÖHLER, A. (2007). "Innovación Docente y Software Libre”. II Encuentro sobre Innovación en Docencia Universitaria. Universidad de Alcalá.

ORTEGA NÚÑEZ, M.A., GARCÍA DÍAZ, P., DÍEZ-PASCUAL, A.M. (2016) El proceso de la innovación en el contexto de la educación superior universitaria. Madrid, LDL. ISBN : 978-84944970-5-6.

(cc) EY-NC-ND 2017, UniversitatPolitècnica de València 